\title{
Temperature identification based on pointwise transient measurements
}

\author{
A. Nassiopoulos \& F. Bourquin \\ French Public Works Research Laboratory (LCPC), Paris, France
}

\begin{abstract}
The inverse problem of temperature identification based on discrete transient measurements is considered. An iterative procedure combining the conjugate gradient algorithm with Tikhonov regularization is used to determine unknown boundary heat fluxes. We show numerically and theoretically that the choice of an $H^{1}$-type space for the minimization gives much better results than a $L^{2}$-type one. A dual formulation of the problem coupled with a model reduction method is used to construct a fast and accurate algorithm suited for real time computations in the three-dimensional case.

Keywords: temperature reconstruction, inverse heat conduction, optimal control theory, adjoint method.
\end{abstract}

\section{Introduction}

Various methods for damage detection and structural health monitoring exist. Among them, the output-only techniques consist in identifying the low frequency spectrum of a given structure in order to detect modifications with respect to the non-damaged reference state. However, in civil engineering, thermal loading due to environmental factors induces mechanical stress on structures and can cause eigenfrequency shifts of much larger order of magnitude than those caused by structural damage [1]. Thus, the knowledge of the exact thermal state of a given structure and its effects on vibrational properties appears as a bottleneck in this field [2].

The need for temperature identification arises from the partial information on the thermal state of a structure one can get. The most commonly used temperature sensors can only provide local or pointwise measurements. In addition, some parts of a structure can simply be inaccessible to direct measurements. This is the case 
in civil engineering structures, although the number of sensors that equip modern ones is rapidly increasing. The thermal state at any point of a given structure can only be obtained through a suitable reconstruction procedure. Among possible methods, one will eliminate Kalman filtering techniques in continuous time which would lead to the solution of Riccati equations of great dimension, and that seems inappropriate without preliminary reduction.

The temperature identification problem can be seen as an inverse problem consisting in inverting the heat equation. This class of problems are in general mathematically ill-posed and regularization techniques are needed to solve them. They have been studied extensively in literature during the last few decades together with many techniques for their numerical computation [3]. Inverse problems concerning heat conduction have been the topic of numerous works due to the wide range of applications that are concerned [4-7]. A review of literature can be found in [8-10].

This paper deals with the inverse problem of temperature identification based on discrete measurements. In view of easy implementation in general purpose finite element software, the approach is that of optimal control theory [11]. The problem is written in a least squares setting. The adjoint technique $[12,13]$ is used to determine the gradient of an error functional together with Tikhonov regularization [3]. When standard gradient-based algorithms are put to work, each computational step entails solving the same forward heat equation with changing data. A dual formulation of the problem and a model reduction technique are put to work to obtain a high speed accurate algorithm suited for real-time applications.

\section{Problem statement}

Consider a solid in a multidimensional domain $\Omega$ with boundary $\partial \Omega$. Assuming absence of internal heat sources and zero initial temperature, the temperature field inside the solid is given by the heat equation:

$$
\begin{cases}\rho c \frac{\partial \theta}{\partial t}-\operatorname{div}(K \operatorname{grad} \theta)=0 & \text { in } \Omega \times[0, T] \\ (K \operatorname{grad} \theta) \cdot \vec{n}+\alpha \theta=\Phi & \text { on } \partial \Omega \times[0, T] \\ \theta(x, 0)=0 & \text { in } \Omega\end{cases}
$$

Here, $\Phi(x, t)=g(x, t)+\alpha \theta_{\text {ext }}$ where $g$ denotes an inward heat flux with FourierRobin conditions and $\theta_{\text {ext }}$ is the external temperature, $\vec{n}$ is the outwards normal vector on the boundary, $x \in \Omega$ is the space variable, $t \in[0, T]$ the time variable, $\rho$ the mass density, $c$ the heat capacity and $K$ the scalar conductivity coefficient of the material taken to be homogeneous and isotropic for the sake of simplicity.

Assume that $m$ sensors are available inside the structure at locations $x_{k}, k=$ 1..m. They deliver the data $\left\{\theta_{k}^{d}(t)\right\}_{k=1}^{m}, t \in[0, T]$. Based on these measurements, the aim is to reconstruct the temperature field over the time interval $[0, T]$, focusing on the accuracy of the temperature field reconstruction at the final instant $T$. 
One way to achieve this is to seek the boundary flux $\Phi$ responsible for the measurements.

The problem of recovering the boundary condition $\Phi$ can be viewed as an operator inversion problem. Consider operator $T$ defined by

$$
\begin{aligned}
T: & U \rightarrow M \\
& T \Phi=\left\{\theta\left(x_{k}, t\right)\right\}_{k=1}^{m}
\end{aligned}
$$

where $\theta(x, t)$ is the temperature field verifying the heat equation (1). In the above, $U$ is the control space ( $\operatorname{such}$ as $\Phi \in U)$ and $M=L^{2}([0, T])^{m}$ is the measurements space (such that $\left\{\theta_{k}^{d}(t)\right\}_{k=1}^{m} \in M$ ). Note that we have not explicitly defined so far the nature of space $U$ as this will be the object of section 3. Under these notations, the temperature reconstruction problem consists in finding $\Phi$ such that

$$
T \Phi=\left\{\theta_{k}^{d}\right\}_{k=1}^{m}
$$

For the sake of simplicity, only the case of the boundary condition $\Phi$ reconstruction is considered in this paper. It is possible to extend all that follows to the case where one wants to reconstruct a couple of functions $\left\{\theta^{0}, \Phi\right\}$ with $\theta^{0}(x)=\theta(x, 0)$ an unknown initial thermal state in (1).

Due to the smoothing properties of operator $T$, the latter is not invertible and the inverse problem (3) is mathematically ill-posed in the sense of Hadamard. The solution of (3) can only be derived in a least squares sense through a functional minimization procedure. The functional can be for instance a quadratic form of the residual term $E=\theta\left(x_{k}, t\right)-\theta_{k}^{d}(t),(k=1 . . m)$, which measures the distance between the data $\theta_{k}^{d}(t)$ and the values at sensor locations of the temperature field determined by $\Phi$.

$$
\begin{aligned}
J(\Phi) & =\frac{1}{2} \sum_{k=1}^{m} \int_{0}^{T}\left(\theta\left(x_{k}, t\right)-\theta_{k}^{d}(t)\right)^{2} d t+\frac{\epsilon}{2}\|\Phi\|_{U}^{2} \\
& =\frac{1}{2}\left\|T \Phi-\left\{\theta_{k}^{d}\right\}_{k=1}^{m}\right\|_{M}^{2}+\frac{\epsilon}{2}\|\Phi\|_{U}^{2}
\end{aligned}
$$

The last term stands for the Tikhonov regularization, $\epsilon$ being a small coefficient that guarantees numerical stability even with noisy input data. $\|\cdot\|_{U}^{2}$ and $\|\cdot\|_{M}^{2}$ are suitable norms in $U$ and $M$ respectively.

\section{Minimization procedures}

The results of the minimization process strongly depend on the a priori assumptions on $\Phi$ that determine the choice of space $U$. In this section two different possibilities are considered, showing that better results are obtained within the framework of an $H^{1}$-type space. 


\subsection{Minimization in $L^{2}$}

The most natural choice consists in looking for a solution $\Phi$ belonging to the space of square integrable functions, namely $L^{2}(\partial \Omega \times[0, T])$. This space can be equipped with the scalar product:

$$
(u, v)_{U}=\int_{0}^{T} \int_{\partial \Omega} u v d \gamma d t, \forall u, v \in U
$$

The adjoint technique is employed to write $J$ as a quadratic form. To this end, we introduce the adjoint operator $T^{*}$ whenever it exists:

$$
\begin{aligned}
T^{*}: & M \rightarrow U \\
& T^{*}\left\{\vartheta_{k}(t)\right\}_{k=1}^{m}=\varphi(x, t)
\end{aligned}
$$

where $\phi$ is the restriction over $\partial \Omega$ of the so-called adjoint state $p$ :

$$
\begin{cases}-\rho c p_{t}-\operatorname{div}(\operatorname{Kgrad} p)=\sum_{k=1}^{M} \vartheta_{k}(t) \delta_{x_{k}} & \text { in } \Omega \times[0, T] \\ (\operatorname{Kgrad} p) \cdot \vec{n}+\alpha p=0 & \text { on } \partial \Omega \times[0, T] \\ p(x, T)=0 \quad & \text { in } \Omega \\ \varphi(x, t)=p\left(\left.x\right|_{\partial \Omega}, t\right) & \end{cases}
$$

Operators $T$ and $T^{*}$ verify the duality relation which writes

$$
M^{\prime}\left\langle T \Phi,\left\{\vartheta_{k}\right\}_{k=1}^{m}\right\rangle_{M}={ }_{U}\left\langle\Phi, T^{*}\left\{\vartheta_{k}\right\}_{k=1}^{m}\right\rangle_{U^{\prime}}
$$

In the above, $U^{\prime}$ and $M^{\prime}$ are the dual spaces of $U$ and $M$ respectively and angle brackets stand for the duality pairing. To formally prove this relation, one has to rewrite equations (1) and (7) in variational form. After an integration by parts of the time derivative term and combination of the last two expressions, one obtains

$$
\sum_{k=1}^{m} \int_{0}^{T} \theta\left(x_{k}, t\right) \vartheta_{k}(t) d t=\int_{0}^{T} \int_{\partial \Omega} \Phi \varphi d \gamma d t
$$

which is exactly relation (8).

Using (8), the functional $J$ can be written as a quadratic form of $\Phi$ :

$$
\begin{aligned}
J & =\frac{1}{2}\left\|T \Phi-\left\{\theta_{k}^{d}\right\}_{k=1}^{m}\right\|_{M}^{2}+\frac{\epsilon}{2}\|\Phi\|_{U}^{2} \\
& =\frac{1}{2}\left[\|T \Phi\|_{M}^{2}-2\left(T \Phi,\left\{\theta_{k}^{d}\right\}_{k=1}^{m}\right)_{M}+\left\|\left\{\theta_{k}^{d}\right\}_{k=1}^{m}\right\|_{M}^{2}\right]+\frac{\epsilon}{2}\|\Phi\|_{U}^{2} \\
& =\frac{1}{2}(A \Phi, \Phi)_{U}-(\Phi, b)_{U}+c
\end{aligned}
$$

with $A=\mathcal{J} T^{*} T+\epsilon \mathbf{I}, b=\mathcal{J} T^{*}\left\{\theta_{k}^{d}\right\}_{k=1}^{m}$ and $c=\left\|\left\{\theta_{k}^{d}\right\}_{k=1}^{m}\right\|_{M}^{2} \cdot \mathcal{J}$ is the Riesz isomorphism between $U^{\prime}$ and $U$ while I stands for the identity operator. It follows 
directly that the gradient of $J$ is given by:

$$
\nabla J(\Phi)=\mathcal{J} T^{*}\left(T \Phi-\theta_{k}^{d}(t)\right)+\epsilon \Phi
$$

This expression means that the gradient of the functional is the restriction on the boundary $\partial \Omega$ of the adjoint field obtained by (7) with a source term $\left\{\vartheta_{k}(t)\right\}_{k=1}^{m}$ equal to the residual $E=\left\{\theta\left(x_{k}, t\right)-\theta_{k}^{d}(t)\right\}_{k=1}^{m}$.

This method of obtaining the gradient allows for a gradient-type method to be used. Each iteration will consist of a computation of the direct equation (1), followed by a computation of the adjoint equation (7) with $\left\{\vartheta_{k}(t)\right\}_{k=1}^{m}=E$. The gradient will then be given by $\left.p\right|_{\partial \Omega}+\epsilon \Phi$. Here, the classical conjugate gradient algorithm was used giving very satisfactory results [7].

Note that equations (1) and (7) have the same structure, so that the same numerical procedure can be used to solve them. The whole algorithm can thus be easily implemented in any classical general purpose scientific software, this being one of the main advantages of the method.

The results below show the disadvantages of the approach described so far. They concern a one-dimensional beam of length $L$ to which a given flux is prescribed at each end. All material constants are set to 1 . Some direct simulations with an arbitrary flux input give measurements on sensors located at $\frac{L}{5}, \frac{L}{2}$ and $\frac{4 L}{5}$ respectively. These measurements are then used to simulate the reconstruction algorithm.

The model is discretized with P1 finite elements and an implicit Euler scheme is used for time integration. In figure 1, the reconstructed flux (dotted line) is compared to the prescribed one (solid line). The flux is well reconstructed on almost the entire time interval, but the accuracy of the results drops near the final instant: the curve exhibits some oscillations and moves away from the target curve to reach zero at $t=T$. As a consequence, the reconstruction of temperature field is of acceptable accuracy far from $t=T$, while the reconstruction of $\theta(x, T)$, which is the most interesting output in view of applications, is very unsatisfactory. This phenomenon is due to the property assigned to the adjoint field $p$ to be null at $t=T$ (equation (7)) and is well known in the literature $[8,13]$.

This final condition on $p$ has to be prescribed in order to verify relation (8). An alternative definition of the adjoint field can be considered, but this implies that the space $U$ has also to be modified. This is the topic of the next section.

\subsection{Minimization in $H^{1}$}

A change of framework for the minimization procedure overcomes this final instant problem. It consists in choosing an $H^{1}$-type space instead of the $L^{2}$-type one.

Take $U=H^{1}\left([0, T], L^{2}(\partial \Omega)\right)$ with the scalar product

$$
(u, v)_{U}=\int_{0}^{T} \int_{\partial \Omega} u v d \gamma d t+\int_{0}^{T} \int_{\partial \Omega} \partial_{t} u \partial_{t} v d \gamma d t, \quad \forall u, v \in U
$$



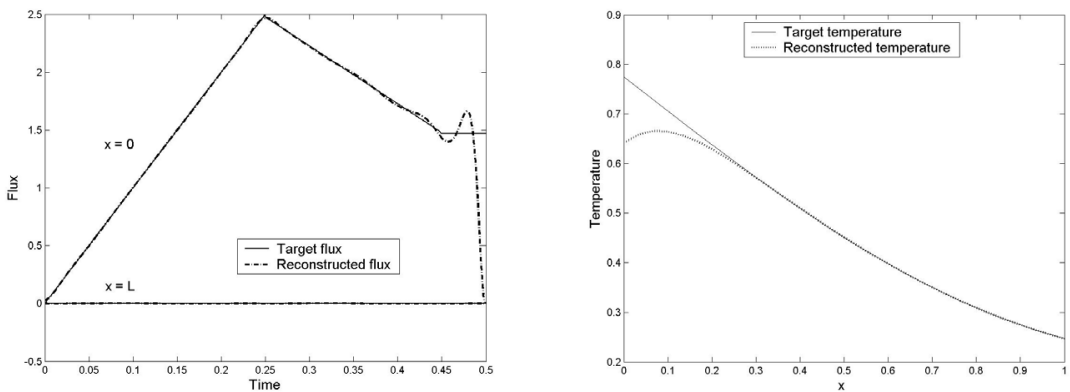

Figure 1: Reconstruction in $L^{2}$ : flux (left) and final temperature reconstruction (right).

In order to verify relation (8), the operator $T^{*}$ has to be defined in a different manner. One can easily verify that if $\varphi$ in (6) is defined by

$$
\int_{0}^{T} \phi p d t=\int_{0}^{T} \phi \varphi d t+\int_{0}^{T} \partial_{t} \phi \partial_{t} \varphi d t, \quad \forall \phi \in H^{1}([0, T] \times \partial \Omega)
$$

where $p$ verifies (7), then operator $T^{*}$ verifies again the duality relation (8) with the new choice of $U$. As can be seen, the computation of $T^{*}$ needs one more step involving the solution of (12) with $p$ known. This last equation is of the form $L(\phi)=a(\varphi, \phi), \forall \phi, a(\cdot, \cdot)$ being a quadratic and $L(\cdot)$ a linear form. It can be easily implemented with the finite element method.

The great advantage of this is that the end condition on $\varphi$ with respect to time is not a Dirichlet one anymore. As a consequence, the value of $\Phi$ at $t=T$ is not fixed and the accuracy of reconstruction near the final instant is much better as can be seen in figure 2 corresponding to the same case as previously. The curves of reconstructed and target final temperatures are identical.
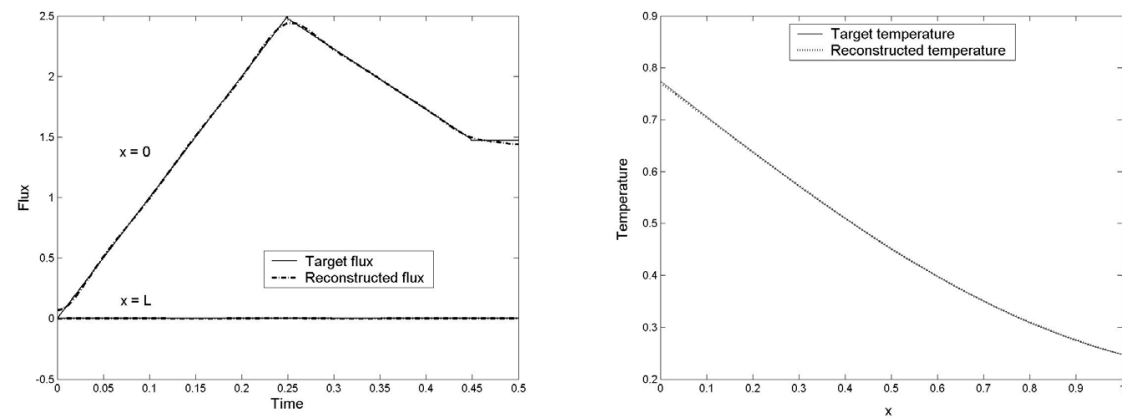

Figure 2: Reconstruction in $H^{1}$ : flux (left) and final temperature reconstruction (right). 


\section{Dual formulation}

Consider the self-adjoint operator $T T^{*}$. Consider next the problem of finding $\left\{X_{k}(t)\right\}_{k=1}^{m} \in M$ such that $T T^{*}\left\{X_{k}(t)\right\}_{k=1}^{m}=\left\{\theta_{k}^{d}(t)\right\}_{k=1}^{m}$. If we set $\Phi=$ $T^{*}\left\{X_{k}(t)\right\}_{k=1}^{m}$, then solving (3) is equivalent to finding $\left\{X_{k}(t)\right\}_{k=1}^{m} \in M$ such that

$$
T T^{*}\left\{X_{k}(t)\right\}_{k=1}^{m}=\left\{\theta_{k}^{d}(t)\right\}_{k=1}^{m}
$$

This new problem is called the dual of (3). It exhibits an ill-posed nature and some form of regularization is needed. Applying Tikhonov regularization, one will consider the problem

$$
T T^{*}\left\{X_{k}(t)\right\}_{k=1}^{m}+\epsilon\left\{X_{k}(t)\right\}_{k=1}^{m}=\left\{\theta_{k}^{d}(t)\right\}_{k=1}^{m}
$$

Due to the self-adjoint nature of $T T^{*}$, the latter is equivalent to minimizing the dual functional $J^{\prime}$ :

$$
J^{\prime}=\frac{1}{2}\left(A\left\{X_{k}(t)\right\}_{k=1}^{m},\left\{X_{k}(t)\right\}_{k=1}^{m}\right)_{M}-\left(\left\{X_{k}(t)\right\}_{k=1}^{m}, b\right)_{M}
$$

with $A=T T^{*}+\epsilon \mathbf{I}$ and $b=\left\{\theta_{k}^{d}(t)\right\}_{k=1}^{m}$.

The dual formulation exhibits many features of interest. Firstly, the problem is set over space $M$ which is of much smaller size than the corresponding space $U$. As a consequence, fewer search directions are involved in the minimization procedure and the conjugate gradient method is expected to converge much faster. Secondly, all scalar products that need to be computed are scalar products in $M$ rather than in $U$ so that the complexity of the algorithm depends more on the number of sensors than on the size of domain $\Omega$. This new formulation results in dramatic reduction of computational costs and in greater adaptability to different geometries. Furthermore, it allows some parallelization of the algorithm, this issue being however beyond the scope of this paper.

\section{Model reduction}

We introduce two distinct function bases $\left\{\xi_{i}\right\}_{i=1}^{\infty},\left\{\chi_{i}\right\}_{i=1}^{\infty}$ that span $H^{1}(\Omega)$, and a third function basis $\left\{\eta_{i}\right\}_{i=1}^{\infty}$ that spans the space $L^{2}(\partial \Omega)$ such that we can approximate the fields $\theta, p$ and $\Phi$ by a reduced order linear combination:

$$
\begin{aligned}
\theta & \sim \theta_{r}(x, t)=\sum_{i=1}^{\ell} \alpha_{i}(t) \xi_{i}(x) \\
p & \sim p_{r}(x, t)=\sum_{i=1}^{\tilde{\ell}} \beta_{i}(t) \chi_{i}(x) \\
\Phi & \sim \Phi_{r}(x, t)=\sum_{i=1}^{\tilde{\ell}} \gamma_{i}(t) \eta_{i}(x)
\end{aligned}
$$

These approximations result in a model reduction that enables a considerable speed-up of the computational procedure, since the size of the systems resulting of 
the finite element discretization of (1) and (7) only depends on the three parameters $\ell, \check{\ell}$ and $\tilde{\ell}$. Under these notations, the objective of reconstruction is to recover the modal coefficients $\gamma_{i}(t), i=1 . . \tilde{\ell}$ of the approximate flux $\Phi_{r}$. The quality of the results depends on the accurate description of the actual variables $\theta, p$ and $\Phi$ by the approximate ones. Another crucial point is the choice of the basis functions in order to ensure observability and controllability of the physical system.

\section{Numerical test case}

Hereafter is shown a numerical example on a three-dimensional case based on this model reduction. A solid of dimensions $L_{x}=1, L_{y}=0.5$ et $L_{z}=0.4$ and material properties all set to 1 is subject to an unknown heat flux on the face of coordinate $x=0$ and zero heat fluxes on all other faces. All boundary conditions are of the Fourier-Robin type. Four sensors are located at points $(0.1,0.4,0.1)$, $(0.1,0.1,0.2),(0.2,0.1,0.3)$ and $(0.2,0.4,0.4)$.

Like in the previous $1 \mathrm{D}$ example, a direct simulation with a prescribed flux on face $x=0$ gives measurements that are taken afterwards as input data for a simulation of the reconstruction algorithm. The prescribed flux has the form shown on figure 3 and can be decomposed into two functions $\eta_{1}(x)$ and $\eta_{2}(x)$ with arbitrary time variation so that $\Phi=\gamma_{1}(t) \eta_{1}(x)+\gamma_{2}(t) \eta_{2}(x)$. Here, $\eta_{1}(x)$ and $\eta_{2}(x)$ are the first two eigenvalues of the Laplace operator on a rectangle with Neumann boundary conditions.
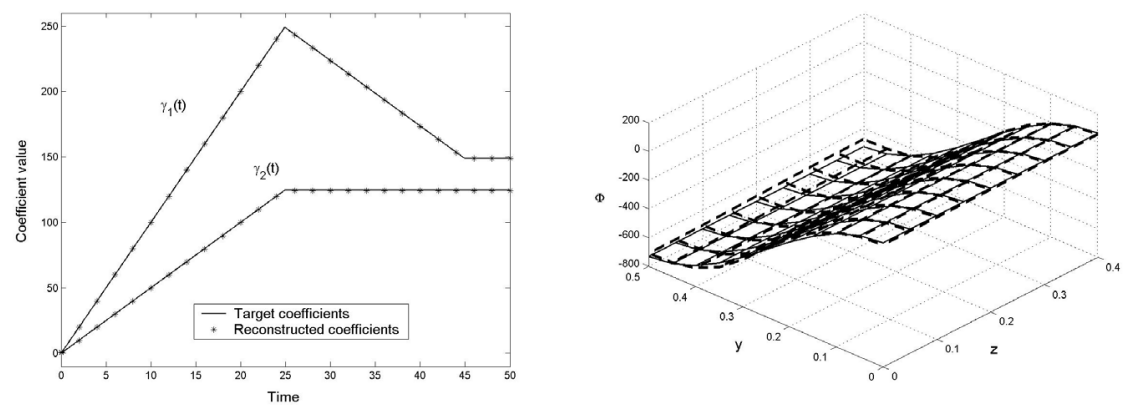

Figure 3: Reconstruction of flux coefficients $\gamma_{1}(t)$ and $\gamma_{2}(t)$ (on the left); prescribed (solid line) and reconstructed (dotted line) flux on face $x=0$ (on the right).

Figure 3 shows the very accurate reconstruction of the two corresponding coefficients $\gamma_{1}(t)$ and $\gamma_{2}(t)$. A very accurate reconstruction of the different components $\alpha_{i}$ of the temperature field obtained under this flux input was also observed. Figure 4 shows the isovalues of the final temperature on two sections on the xy-direction and the isovalues of the difference between reconstructed and target fields: there is 
a ratio of $10^{4}$ between the corresponding orders of magnitude. The reconstruction took 4.4s CPU for 20 iterations.
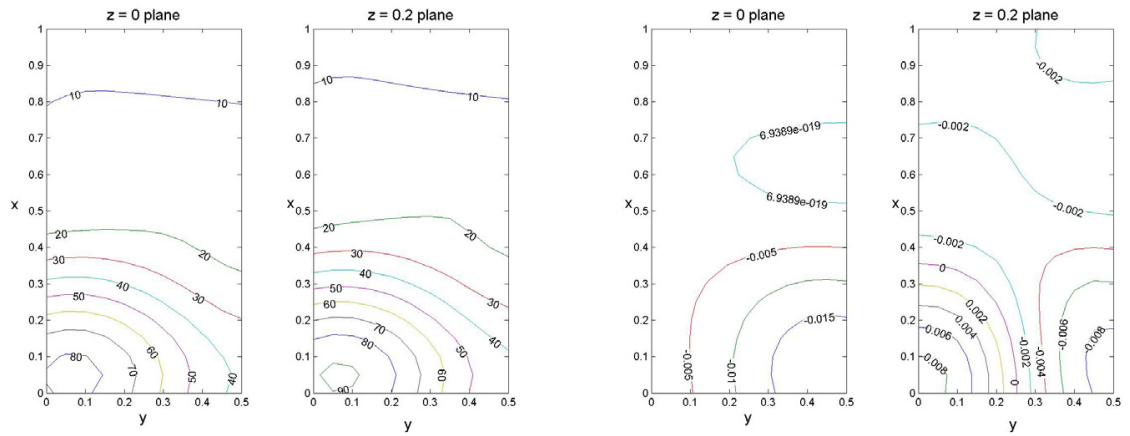

Figure 4: Isovalues of the temperature field on planes $z=0$ and $z=0.2$, at $t=T$ : target field (left); difference between target and reconstructed fields (right).

\section{Conclusions}

The adjoint technique for the minimization of a least squares error functional has been applied with success to the problem of temperature reconstruction based on pointwise measurements. The use of the $H^{1}$ space has proved to be the best-suited framework for the minimization procedure, and has permitted us to overcome the final instant problem of the classical technique. The dual formulation of the problem and the model reduction using well chosen basis functions enables us to carry very fast computations with great accuracy. The algorithm can thus be adapted for the real time monitoring of structures. In such a case, the reconstruction procedure can be carried out at given intervals with overlapping time domains: the initial temperature condition for each computation will be known from the previous reconstruction computation and added to the reconstructed field. The entire procedure could be compared with an observer of great efficiency and speed.

\section{References}

[1] Farrar, C., Hemez, F., Shunk, D., Stinemates, D. \& Nadler, B., A review of shm literature: 1996-2001. Los Alamos National Laboratory Internal Reports, 2003.

[2] Kullaa, J., Elimination of environmental influences from damagesensitive features in a structural health monitoring system. Proceedings of the First 
European Workshop on Structural Health Monitoring, Paris, July 10-12, 2002, Onera. DEStech Publications, pp. 742749, 2002.

[3] Engl, H.W., Hanke, M. \& Neubauer, A., Regularization of Inverse Problems. Kluwer Academic Publishers, 1994.

[4] Beck, J.V., Blackwell, B. \& Haji-sheikh, A., Comparison of some inverse heat conduction methods using experimental data. Int J Heat Mass Transfer, 39(17), pp. 36493657, 1996.

[5] Neto, A.J.S. \& Özisik, M.N., Two-dimensional heat conduction problem of estimating the time-varying strength of a line heat source. J Appl Phys, 71(11), pp. 53575362, 1992.

[6] Videcoq, E. \& Petit, D., Model reduction for the resolution of multidimensional inverse heat conduction problems. Int J Heat Mass Transfer, 44, pp. 18991911, 2001.

[7] Prudhomme, M. \& Nguyen, T.H., Fourier analysis of conjugate gradient method applied to inverse heat conduction problems. Int J Heat Mass Transfer, 42, pp. 44474460, 1999.

[8] Alifanov, O.M., Inverse Heat Transfer Problems. Springer-Verlag, New York, 1994.

[9] Beck, J.V., Blackwell, B. \& Clair, C.S., Inverse heat conduction, illposed problems. Wiley Interscience, New York, 1985.

[10] Özisik, M.N. \& Orlande, H.R.B., Inverse Heat Transfer. Taylor and Francis, 2000.

[11] Lions, J.L., Optimal control of systems gouverned by PDEs. Dunod, 1968.

[12] Jarny, Y., Özisik, M.N. \& Bardon, J.P., A general optimization method using adjoint equation for solving multidimensional inverse heat conduction. Int $J$ Heat Mass Transfer, 34(11), pp. 29112919, 1991.

[13] Huang, C.H. \& Wang, S.P., A three-dimensional inverse heat conduction problem in estimating surface heat flux by conjugate gradient method. Int J Heat Mass Transfer, 42, pp. 33873403, 1999. 